Review

\title{
Readying Directed Self-Assembly for Patterning in Semi-Conductor Manufacturing
}

\author{
Roel Gronheid ${ }^{1, *}$, Paulina Rincon Delgadillo ${ }^{1,2,3}$, Arjun Singh ${ }^{1,2}$, Todd R. Younkin ${ }^{4}$, Safak Sayan $^{4}$, \\ Boon Teik Chan ${ }^{1}$, Lieve Van Look ${ }^{1}$, Joost Bekaert ${ }^{1}$, Ivan Pollentier ${ }^{1}$, Paul F. Nealey ${ }^{3}$ \\ ${ }^{1}$ Imec vzw, Kapeldreef 75, B-3001 Leuven, Belgium \\ ${ }^{2}$ Katholieke Universiteit Leuven, Department of Electrical Engineering (ESAT), \\ Kasteelpark Arenberg 10, B-3001 Leuven, Belgium \\ ${ }^{3}$ Institute for Molecular Engineering, University of Chicago, 5747 South Ellis Avenue, Jones 217, Chicago, \\ IL 60637, USA \\ ${ }^{4}$ Intel assignee to imec \\ *roel.gronheid@imec.be
}

\begin{abstract}
In recent years Directed Self-Assembly (DSA) has come up as a strong candidate technology for advanced lithography. DSA is a complementary technology that is used to enhance established projection lithography. In this paper, an overview is given of activities at imec that are driving towards readying DSA technology for implementation into semiconductor manufacturing. Flows for line/space and contact hole pattern formation based on chemo- as well as grapho-epitaxy are available and used for understanding process sensitivities, evaluation of defect densities and the demonstration of integration approaches. The state-of-the-art of the various process flows is discussed along with the primary outstanding issues.
\end{abstract}

Keywords: directed self-assembly, grapho-epitaxy, chemo-epitaxy, lamellar phase block copolymers, cylindrical phase block copolymers, metrology

\section{Introduction}

Advanced patterning using Directed SelfAssembly (DSA) of Block Co-Polymers (BCP) has gained an enormous increase of interest over the past four years [1]. It has advanced from an academic novelty to a patterning technique that is considered for near term semi-conductor applications [2] as well as in other fields of the micro-electronics industry [3]. This review will describe the recent progress that has been made at imec in readying DSA technology for introduction for IC manufacturing.

BCPs have the ability to form dense arrays of periodic structures of diverse shapes on the nanoscale. This review will focus on the specific case of di-block copolymers (which are the simplest of the BCP systems). In this case only five, relatively simple structures may be formed upon annealing of these materials. The type of structure that is formed is, in principle, independent of the chemistry of the blocks of the BCP material, and solely determined by the relative volume fraction of the two blocks. In the order of increasing volume fraction of one block with respect to the other, face-centered cubically packed spheres, body-centered cubically packed spheres, hexagonally packed cylinders, ordered bicontinuous double diamond and lamellar phase may be distinguished, respectively $[4,5]$.

Similar structures to the ones that are formed in the bulk may also be formed in these materials when they are spin-coated as thin films on a carrier substrate. For manufacturing purposes, primarily the cylindrical and lamellar phases are of interest. The lamellar phase may be used for fabrication of line/space type patterns when the structures are oriented perpendicularly with respect to the surface that they are patterned on. The cylindrical phase may be used for hole patterns in case of perpendicular orientation and for line/space type patterns in the case of horizontal orientation. Even though the latter approach is actively explored for 


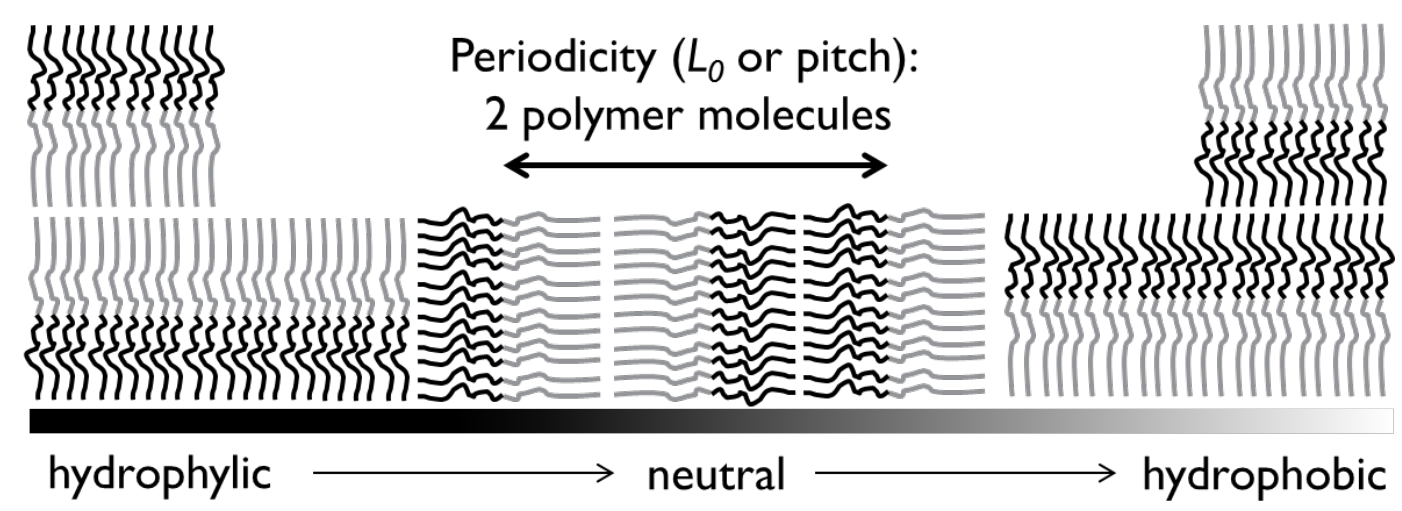

Figure 1. Schematic representation of how BCP molecules with a hydrophilic (black) and hydrophobic (grey) segment align on a surface depending on the surface hydrophobicity. It should be noted that the discrete topography steps that are typically encountered in case of perpendicular orientation of the polymer molecules may have a height of $1 * \mathrm{~L}_{0}$ or $0.5 * \mathrm{~L}_{0}$ depending on the surfacting properties of the two polymer segments.

patterning purposes [6] it will be out of scope for this paper.

Aligning the BCP-based patterns with respect to each other and positioning them in the desired location is crucial for most manufacturing applications. Within a short time span, various groups came up with different approaches to achieve this orientation control [7-9]. These were the first demonstrations of what today is known as DSA. The final BCP patterns that will be obtained are determined by a fine interplay of $\mathrm{BCP}$ properties on the one hand, and geometry as well as surface chemistry of the pre-pattern on the other. As will be demonstrated in the following sections, the fabrication of the optimal pre-pattern is at least as important as the optimization of the BCP material for achieving high quality DSA patterns.

In DSA, in contrast to traditional optical lithography, the polymeric material contains information on the final target structures. The polymers have an intrinsic natural periodicity $\left(\mathrm{L}_{0}\right)$, which will determine the critical dimension (CD) of the resulting structures, as $\mathrm{CD}$ is more or less fixed at $\sim 0.5 \mathrm{~L}_{0}$. This dimensional control can be employed to improve (local) CD uniformity or in more extreme cases even repair merging prepattern structures [10]. Even more compelling, DSA may be used to increase pattern density. When the pre-pattern dimension matches an integer multiple of $\mathrm{L}_{0}$, multiple periods of the $\mathrm{BCP}$ may assemble in between the guiding structures, resulting in pattern densities greater than the resolution provided by current optical techniques $[11,12]$. The geometrical properties of the prepattern that is used to guide the BCP structure thus need to be matched to the dimensional properties of the BCP (commensurability).

The rate of advancement of the technology from the first demonstration to making these types of flows compatible with full-wafer processing has been remarkable. Especially over the past 3-4 years, the global efforts from semi-conductor equipment and material suppliers have increased significantly. Nevertheless, multiple outstanding issues still exist before the technique may be widely adopted. The primary points of attention include defectivity, DSA-specific metrology requirements (including 3D metrology of the DSA structures), pattern placement accuracy (part of which appears as pattern roughness), and DSA-friendly design decomposition. In the DSA projects at imec all of these aspects are investigated. The work described in this review will mainly focus on the defectivity and metrology aspects.

DSA is recognized to be a technology with great potential for extendibility. Patterns with half pitches as small as $\sim 3 \mathrm{~nm}$ have been demonstrated [13]. However, the main work horse for DSA development today is poly(styrene- $b$ methylmethacrylate) (PS- $b$-PMMA), which has a resolution that is limited to $\sim 12 \mathrm{~nm}$ half pitch. The properties of this material allow for easy processing, since the surface energies of the PS and PMMA are sufficiently close. This allows that phase separation is obtained by thermal annealing in inert atmosphere such as $\mathrm{N}_{2}$ or vacuum without formation of a surface wetting layer by either of the blocks. However, in order to further extend the DSA technology beyond the resolution that is provided by the PS- $b$-PMMA material, so-called high- $\chi$ materials will be required. The Flory- 
Huggins interaction parameter $(\chi)$ describes the degree to which the two blocks 'repel' or attract each other. PS- $b$-PMMA has a relatively low $\chi$ parameter of $\sim 0.04$ at the annealing temperature [14]. High- $\chi$ materials in many cases are more difficult to process, due to differences in the surface energies of the two blocks. Use of a topcoat [15] or solvent annealing [16, 17] may be required to achieve the desired structure orientation. The development of these high- $\chi$ materials is currently a topic of intensive research at material suppliers, but they still are in a much earlier phase of readiness for implementation. The work that will be described in this review will all be focused on the PS- $b$-PMMA BCP materials.

This paper will discuss various flows, as implemented at imec, through which DSA may be used. Recent advancements in performance improvements will be highlighted.

\section{Materials characterization for DSA}

At least two classes of materials (in most cases both polymeric) are required for the common DSA implementation approaches. Next to the BCP material itself, in most cases also a so-called 'neutral layer' is required. This material serves to condition the surface energy of the substrate such that the desired wetting properties of the $\mathrm{BCP}$ material on the substrate are obtained. An untreated substrate will, in most cases, preferentially wet one of the blocks of the BCP. For instance, if the substrate is relatively polar (e.g. native oxide of a silicon wafer), the more polar block of the BCP material will preferentially wet this surface (Figure 1, left). In this case the polymer molecules will orient perpendicular to the surface and the resulting structures (lamellae or cylinders) from the BCP will form parallel to the substrate. When making the surface less polar, the driving force for this preferential wetting disappears until at a certain point it becomes sufficiently small and the polymers of both blocks can wet the surface (Figure 1, middle). At this stage the polymer molecules are oriented parallel to the surface and the resulting structures are perpendicular to the surface. When the substrate is made even more apolar, the situation is reversed and the apolar block of the BCP preferentially wets the surface (Figure 1, right). Neutral layer materials may be composed of random copolymers consisting of the same monomer units as used for the BCP material itself. The relative composition may in this case be tuned such that the desired neutral behavior is obtained. However, also other materials, not containing the BCP monomer units, such as spin-on hard mask layers may have tuned surface energies such that they behave as neutral layers.

When neutral materials samples are first introduced in the fab a quick test for checking their neutral behavior in combination with the $\mathrm{BCP}$ material of interest is available. A $300 \mathrm{~mm}$ silicon wafer is first coated and baked with the neutral layer and next with the BCP of interest. This test relies on the fact that in the case of preferential wetting, a uniform film can only be formed if the film thickness (FT) exactly matches an integer multiple of the layer spacing that is inherent to the $\mathrm{BCP}\left(\mathrm{FT}=n \mathrm{~L}_{0}\right)$ for symmetric wetting, or $\mathrm{FT}=$ $(n+1 / 2)^{*} \mathrm{~L}_{0}$ for asymmetric wetting. In other cases, either 'holes' or 'islands' will be formed in the BCP film. This results in micron-size structures in the BCP film after coat and annealing, which can be easily visualized by optical or low magnification (2-10kX) SEM microscopy (Figure 2 , left). In the case of proper neutrality, a uniform, flat film is obtained irrespective of the film thickness and nanometer-sized randomly oriented BCP patterns can be visualized by high magnification (150-300kX) SEM (Figure 2, right).
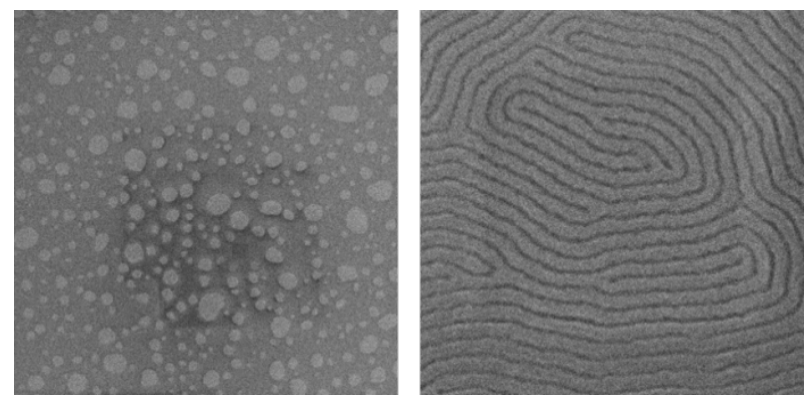

Figure 2. SEM microscope images of the same BCP material on two different surfaces displaying preferential wetting resulting in hole-and-island structures (left) and neutral wetting resulting in fingerprint patterns (right)

Besides the neutral layer material also the properties of the unguided BCP material, such as its $\mathrm{L}_{0}$, should be checked before it is evaluated in a DSA process. The process flow for such an experiment is very similar to that described above for the neutral layer evaluation, except for the fact that in this case a known good neutral layer is used and the final target thickness for the $\mathrm{BCP}$ is applied. In addition to determining the $\mathrm{L}_{0}$ of the 
BCP from the so-called 'fingerprint' SEM images that are obtained from this test, insight in the correlation length or grain size of the selfassembled domains [18] and the roughness of the unguided patterns [19] may be gained.

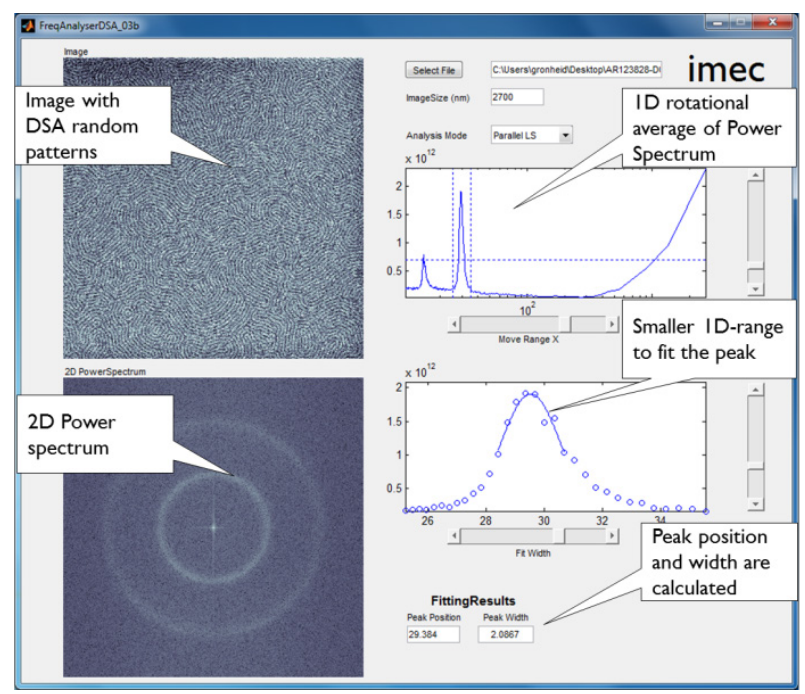

Figure 3. Screenshot of frequency analysis software to determine the BCP natural periodicity from SEM images of unguided $\mathrm{BCP}$ on a neutral surface.

For comparison of $\mathrm{L}_{0}$ from the unguided patterns, Fast-Fourier Transform (FFT) analysis is an effective method. An in-house developed software tool allows quick and easy determination of $\mathrm{L}_{0}$ via this method (Figure 3). The definition of $\mathrm{L}_{0}$ in the case of lamellar phase BCP is straightforward and simply is identical to the pitch of the pattern. In the case of cylindrical phase BCP, some care should be taken. For lithographic purposes, the center-to-center distance of the holes is of primary importance. However, the cylinders prefer a closest packing and will thus form hexagonal arrays. $\mathrm{L}_{0}$ in this case is defined as the pitch of separate rows of holes (Figure 4). On simple geometrical basis, the relation between the center-to-center distance $(a)$ and $\mathrm{L}_{0}$ is given by

$$
a=2 / 3 * L_{0}
$$

\section{Directed self-assembly processes}

Two main approaches for guiding the BCP patterns are distinguished. The first is called grapho-epitaxy, which uses the topography of the pre-pattern to guide the BCP (Figure 5, left). In this case, the prepattern structure itself becomes an integrated part of the final pattern structure. When this pattern is thus used in a next process step, such as further pattern transfer using dry etch, also the pre-pattern must have masking properties for this process step. The second approach is called chemo-epitaxy and uses a pre-pattern that is nominally flat but contains nano-structured surface chemistry variations (Figure 5, right).

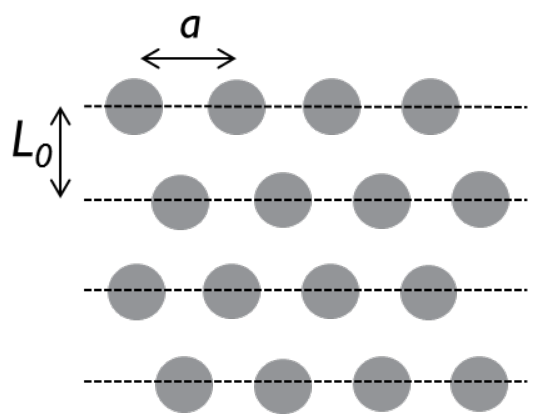

Figure 4. Schematic representation of hexagonally packed cylinders. The natural periodicity $L_{0}$ is defined by the spacing of rows. This defines the center-to-center distance of the holes $a$ (Equation 1).

It should be noted that the separation of DSA processes in grapho- and chemo-epitaxy is artificial and most processes contain aspects of both. For example, the wetting behavior of the $\mathrm{BCP}$ on the sidewalls of the template in a graphoepitaxy process is of great importance in determining the final outcome of the structure. In most chemo-epitaxy processes, some substrate topography is present of which the impact in the DSA pattern formation is not always clear.

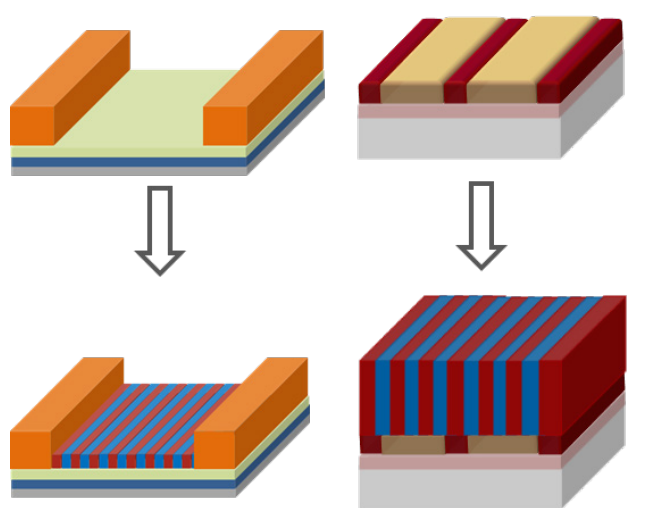

Figure 5. BCP patterns may be guided by topography on the substrate, which is called grapho-epitaxy (left) or by nano-patterned surface energy variations, which is called chemo-epitaxy (right).

Benefits of grapho-epitaxy include potential process simplicity, high potential degree of frequency multiplication and increased flexibility for random placement of structures. Benefits for chemo-epitaxy include process robustness and 


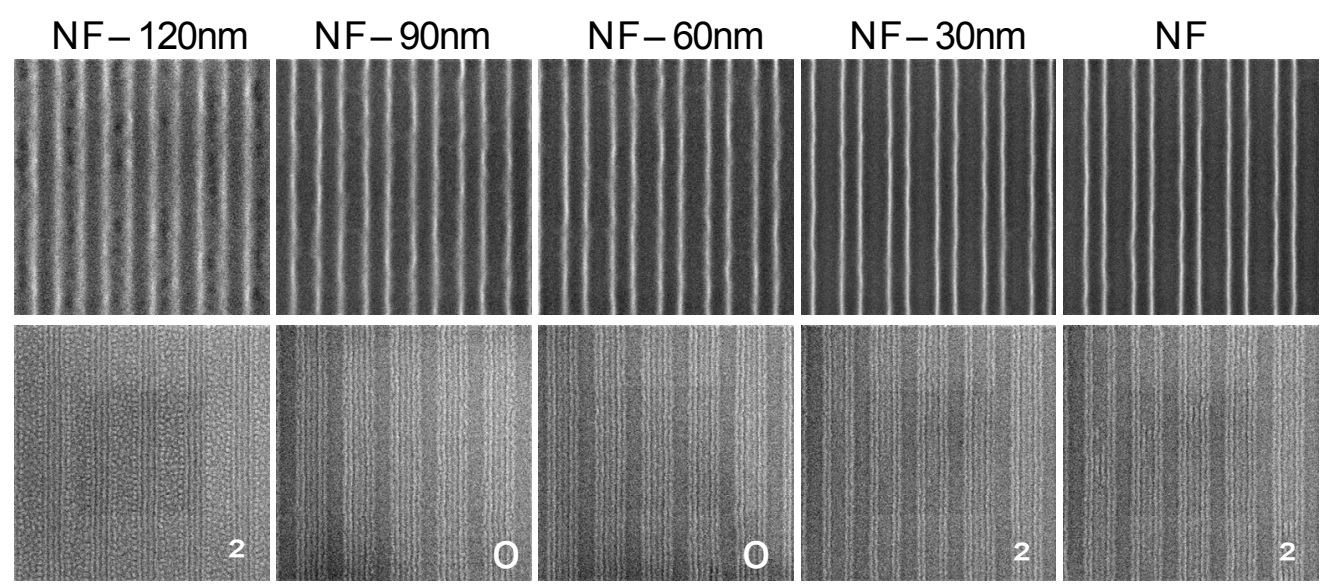

Figure 7. Top-down SEM images through focus at fixed dose for the nominal 150nm space width features for the hardened pre-pattern structures (top) and after BCP $\left(25 \mathrm{~nm} \mathrm{~L}_{0}\right)$ anneal (bottom). In the bottom row the ' $\times$ ' symbol is used to indicate what is judged as poor and 'o' to indicate what is judged as good BCP alignment

avoiding that guide patterns consume surface area (resulting in higher potential for pattern density). Selection of the approach for direction will likely depend on the application.

At imec both grapho- and chemo-epitaxy has been used for implementation of line/space as well as hole patterning (Table 1). The next sections will discuss recent key achievements of these four subprojects.

\begin{tabular}{|l|c|c|}
\hline & Grapho-epitaxy & Chemo-epitaxy \\
\hline Lines/Spaces & VINAIGRETTE flow & LiNe flow \\
\hline Contacts & $\begin{array}{l}\text { 1. EVEREST28 flow } \\
\text { 2. } 1000 \text { ISLANDS flow }\end{array}$ & HONEYCOMB flow \\
\hline
\end{tabular}

Table 1. Available process flows at imec. Line/space as well as contact hole structures are patterned with grapho- as well as chemo-epitaxy.

\subsection{Grapho-epitaxy of line/space patterns}

In 2011, imec started efforts to evaluate DSA for line/space frequency multiplication based on grapho-epitaxy using the dedicated DSA mask code-named 'VINAIGRETTE'. The process flow is schematically depicted in Figure 6 [20]. In this case, a negative tone resist was used, which is hardened in a post-development bake step. The hardening avoids dissolution of the resist patterns in the solvent of the $\mathrm{BCP}$ when this material is applied for spin-coating. Moreover, the hardening avoids reflow during the thermal BCP anneal. Initial process optimization focused on neutral layer material selection, and thickness optimization for guide pattern as well as BCP. Most of this study employed a $25 \mathrm{~nm} \mathrm{~L}_{0} \mathrm{BCP}$ material, resulting in $12.5 \mathrm{~nm}$ line/space patterns. Typical pre-patterns involved $150-200 \mathrm{~nm}$ trenches at $250-300 \mathrm{~nm}$ pitch and were patterned using 0.85NA ArF lithography.

Further studies mainly aimed on determining the sensitivity of the process towards variations in the pre-pattern. It was found that the profile of the pre-pattern had a significant impact on the resulting structure. Interestingly, a slightly sloped profile seemed to be preferred for optimal BCP pattern formation as judged from top-down inspection of the BCP structures after thermal anneal (but before PMMA removal). As a result, the optimal operating point (in terms of both dose and focus) for pre-pattern imaging does not correspond to the optimal operating point for the DSA process (Figure 7).

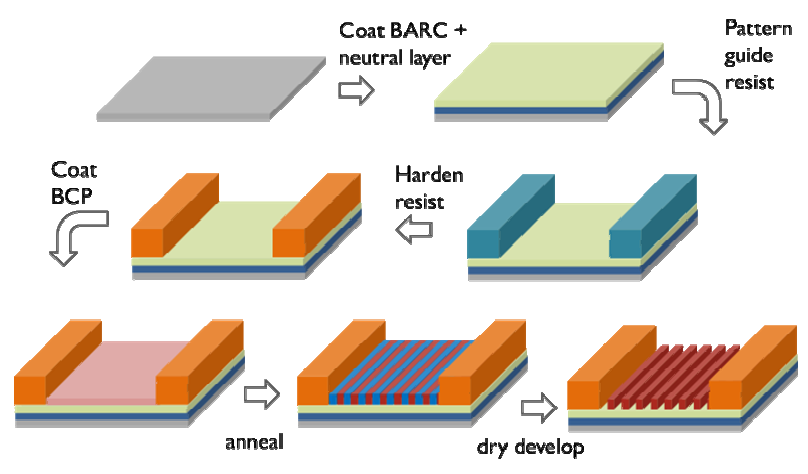

Figure 6. Schematic representation of the VINAIGRETTE grapho-epitaxy flow for line/space patterning.

In addition, it was found that the graphoepitaxy DSA process is very sensitive to imperfections in the pre-pattern. Programmed protrusion defects in the pre-pattern were used to 

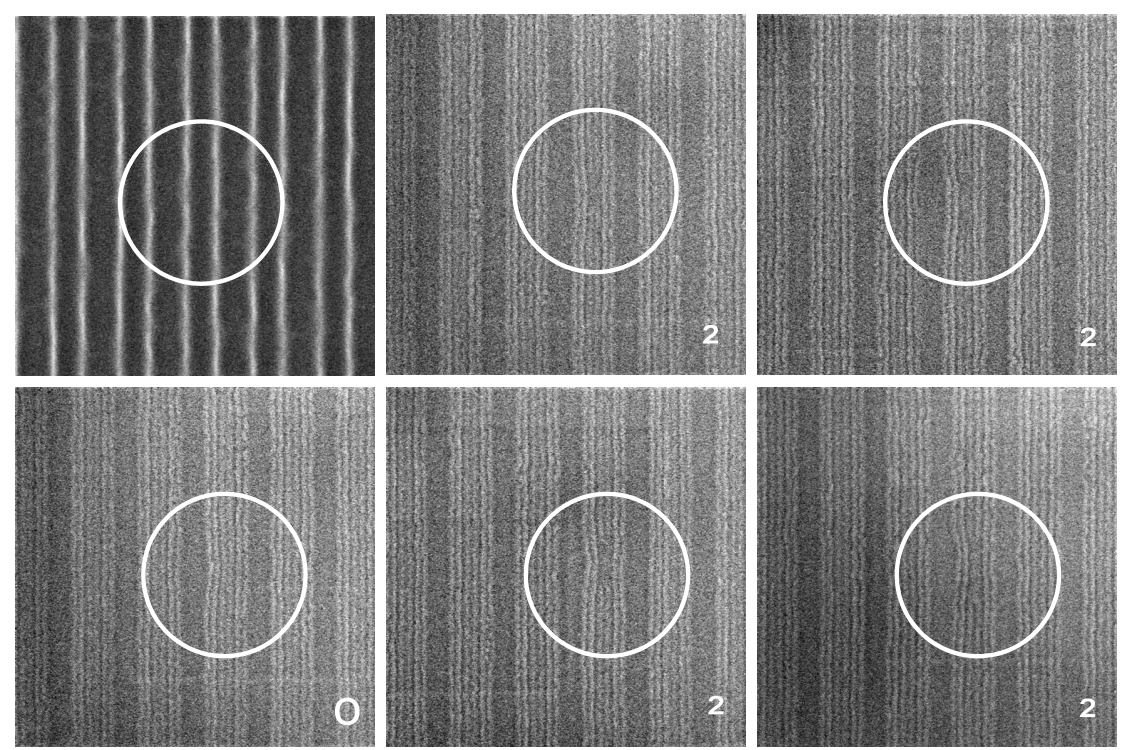

Figure 8. Top-down CD-SEM image of an $8 \mathrm{~nm}$ amplitude protrusion defect (top left) in the pre-pattern and a representative set of samples of this defect location after BCP self-assembly ( $25 \mathrm{~nm}$ L0 periodicity). The exact location of the protrusion defect in the SEM image is indicated by the white circle. The ' $x$ ' symbol is used to indicate what is judged as poor and 'o' to indicate what is judged as good BCP alignment.

determine at what stage these start to impact the $\mathrm{BCP}$ pattern formation. Even though the $6 \mathrm{~nm}$-sized defect in the pre-pattern is barely visible from the top-down SEM image, it already has a relatively high probability of generating impact on the DSA pattern (Figure 8). Protrusion defects of $8 \mathrm{~nm}$ and larger generated DSA perturbations with close to $100 \%$ probability. This study demonstrates that even though relatively 'low-end' optical lithography may be used for achieving the target resolution for the pre-patterns, the requirements on feature edge placement are very strict.

\subsection{Chemo-epitaxy of line/space patterns}

The majority of the work on line/space patterning with chemo-epitaxy has focused on the $14 \mathrm{~nm}$ half pitch Liu-Nealey (LiNe) flow (Figure 9) [21]. A detailed discussion of the process optimization for this flow is published elsewhere in this issue [22]. The primary goal of this work is two-fold. At first, it is determined how process variables impact defect formation in DSA processing. The control that the LiNe flow offers over the geometry as well as the surface properties of the chemical pre-patterns is crucial in this respect [23-26]. Secondly, we intend to demonstrate that DSA can approach defect densities that are sufficiently low for the purpose of semi-conductor manufacturing.

To accelerate the process optimization, efforts on developing novel metrology approaches are undertaken. It should be noted that in contrast to optical lithography, the standard CD measurement is not a good figure of merit for assessing the DSA process quality. Since the $\mathrm{CD}$ of a line structure is mainly determined by the BCP material, it will be barely affected by important process variables such as CD of the pre-pattern. Rather, the degree of alignment is a more valuable measure. SEM is a slow means of determining the degree of alignment. Due to the limited field of view of a typical SEM image, a large number of images per exposed field $(>10)$ needs to be collected to obtain sufficient statistical information. In addition, automated image analysis of the degree of alignment from low magnification SEM images (required for a sufficiently large field of view) is non-trivial.

In order to quickly screen materials and process conditions, an optical technique based on the ASML YieldStar ${ }^{\mathrm{TM}}$ S-200 scatterometer has been developed [27]. This optical metrology technique gives a single number as output. This parameter contains not only information about the degree of ordering but also on other process related aspects, including film thickness, pattern $\mathrm{CD}$, and pitch. The absolute value of the parameter is therefore not informative. Normalization to a known good location on the wafer is typically required to extract meaningful data. The advantage of the technique is that it can quickly provide information of the $\mathrm{BCP}$ pattern quality either before or after 


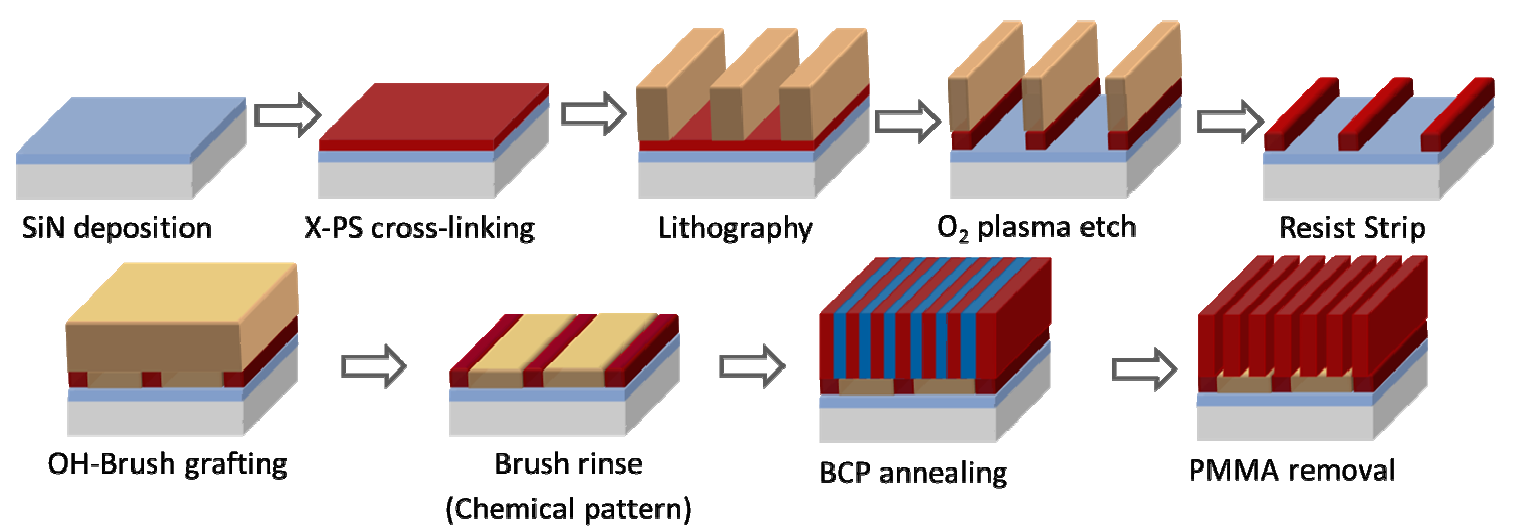

Figure 9. Schematic representation of the LiNe chemo-epitaxy flow for line/space patterning

PMMA removal. Figure 10 demonstrates an example for optimization of the neutral layer composition. Six materials are assessed by their degree of alignment on an focus-exposure-matrix (FEM)-exposed pre-pattern wafer. In such a wafer layout, exposure dose increases from left to right and focus from top to bottom. The match between SEM and optical analysis is very good.

Also LWR requires dedicated metrology development for DSA. In order to properly break down how the final LWR is impacted by the prepattern roughness, BCP materials and the dry development process, it is needed to be able to measure the roughness of guided as well as unguided $\mathrm{BCP}$ structures. For the guided $\mathrm{BCP}$, the standardly available LWR algorithms that are available on state-of-the-art CD-SEM equipment may be used. For the more randomly oriented unguided structures dedicated analysis techniques have been developed [19].

With respect to the second defectivity-related goal, a weekly DSA defect monitor of the LiNe flow is run in the imec $300 \mathrm{~mm}$ facilities. All three materials (XPS guide material AZEMBLYTM NLD-128, neutral brush material AZEMBLYTM NLD-127, and 28nm $\mathrm{L}_{0}$ BCP material AZEMBLYTM PME-312) for the flow are available in gallon quantities and installed on our DSAdedicated TEL CLEAN TRACK ACT ${ }^{\mathrm{TM}} 12$ for inline dispense. The defect monitor serves as a baseline to compare the impact of potential process improvements [28]. When these are identified they are implemented in the monitor resulting in lower baseline defectivity. Figure 11 gives the defect monitoring results over 24 weeks in 2013.

Defect source analysis [29] and SEM-based defect classification are used to identify the primary contributors to the baseline defectivity. Initial high counts were primarily due to 'black spot' defects. These could be traced to originate from the neutral layer material. A re-installation of

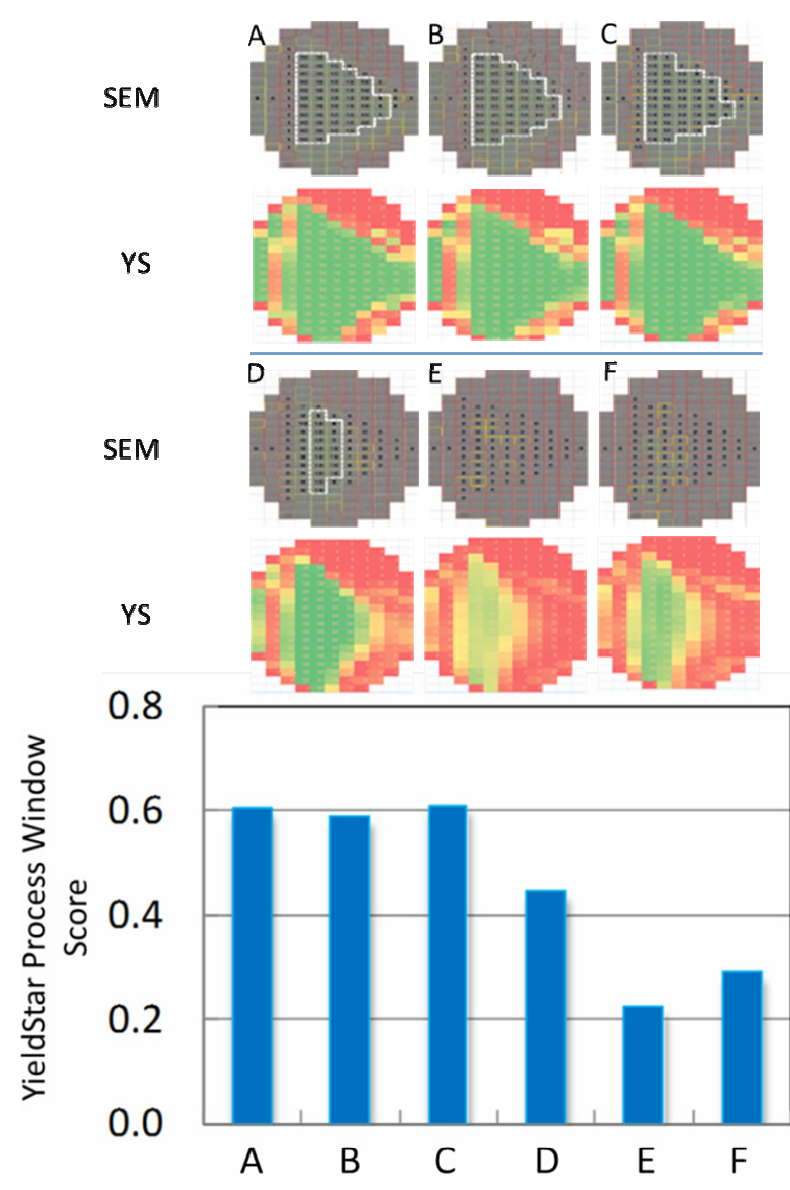

Figure 10. Comparison of process windows as determined from FEM wafer maps for brush formulation A-F. Wafer map of SEM based measurements is each time give on top and of optical metrology on the bottom. The white lines in the SEMbased wafer maps indicate the area of defect-free alignment. The color scales of the optical wafer maps are identical. The bar graph indicates the normalized full wafer average of all optical scores and is indicative of the process window. 


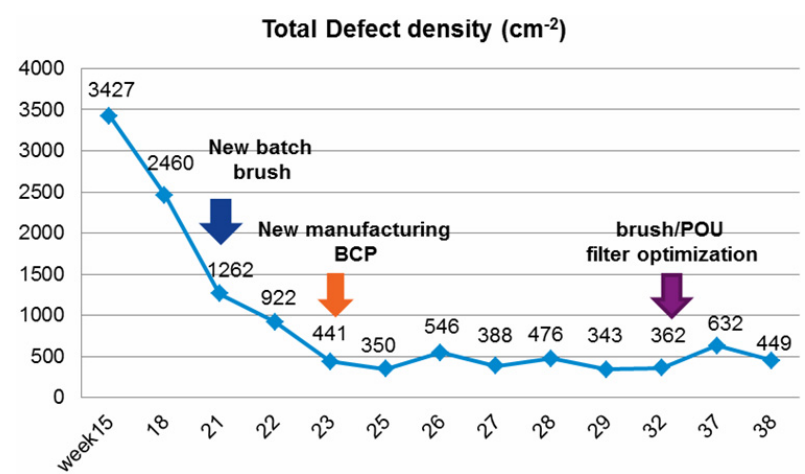

Figure 11. Raw defect trend in the $14 \mathrm{~nm}$ half pitch LiNe flow over a 24 week period.

the neutral layer material solved this problem. Next, gel particles in the BCP created small bridging defects. A new purification approach in the manufacturing of this material at AZ Electronic Materials reduced the occurrence of this type of defects by $>98 \%$. Also collapse type defectivity was tackled. The collapse was mostly seen to nucleate from a particle, which could be traced to stem from the neutral layer material. A new filter material for point-of-use filtration significantly reduced the impact of these defects. After these interventions SEM non-visible (SNV) defects are the primary remaining defect classes. Etch studies have demonstrated that these defects are not caused by buried particles. The SNV classes are thus false defects due to metrology noise. The primary focus is currently thus on improving the signal-to-noise for defect inspection. Nevertheless, the current champion defectivity data at $\sim 130$ defects $/ \mathrm{cm}^{2}$ is still several orders of magnitude removed from manufacturing requirements. It is, however, encouraging to see that the defect types mostly are of particle origin and not caused by the intrinsic thermodynamics of the DSA process. In this sense, the defectivity challenges for DSA are not different from that of other patterning techniques that target the same dimensions. Continued improvements in material and process are thus expected to enable to further drive down the defect performance.

Finally, efforts have been made to transfer the line/space patterns of the LiNe flow into the underlying silicon substrate. This is a typical way in which these patterns may be used for defining the initial Fin layer for FinFET devices. Without using any specific hard mask materials and just using the initial PS patterns on top of the SiN ARC, the $14 \mathrm{~nm}$ half pitch patterns were etched $67 \mathrm{~nm}$ deep into Si (Figure 12). This demonstrate that
$7 \mathrm{~nm}$ node compatible Fin structures can be printed using this process flow.

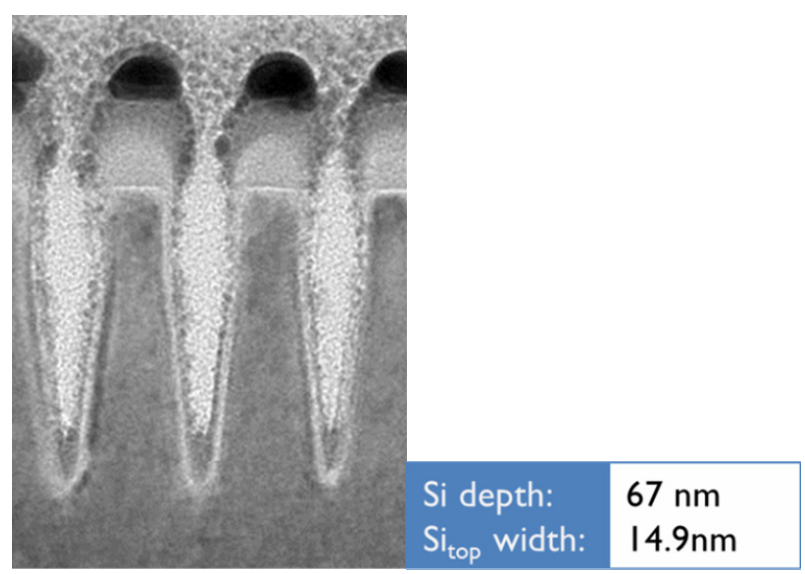

Figure 12. TEM image of $14 \mathrm{~nm}$ half pitch patterns from the LiNe flow transferred $67 \mathrm{~nm}$ deep into $\mathrm{Si}$.

\subsection{Grapho-epitaxy of hole patterns}

The most simple case of DSA with graphoepitaxy to generate hole patterns is single hole shrink. Besides CD shrink, in most cases improved CD uniformity (CDU) of the holes is obtained, since the hole $\mathrm{CD}$ will be determined by the polymer physical dimensions. BCP materials may be used for this approach, but homo-polymer blends have been demonstrated to also be effective for this process when applied on a negative tone photoresist [30]. The use of homo-polymers allows for milder thermal anneal conditions and efficient solvent development to remove the central block after the DSA process. In addition, the CD shrink is less aggressive compared to the $\mathrm{BCP}$ approach.
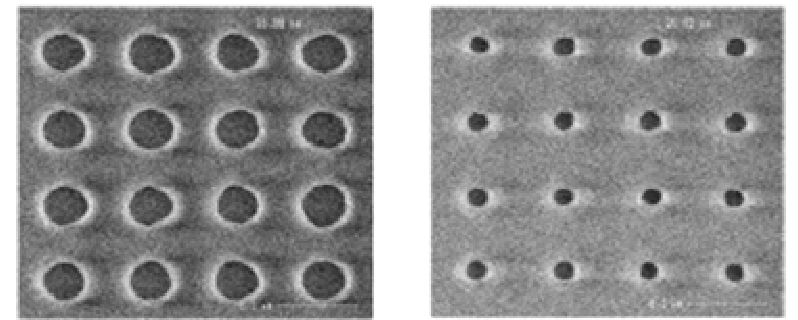

Figure 13. CD in resist (left) is reduced from $56 \mathrm{~nm}$ $(\mathrm{CDU}=4.9 \mathrm{~nm} 3 \sigma)$ to $27 \mathrm{~nm}(\mathrm{CDU}=1.6 \mathrm{~nm} 3 \sigma)$ after homo-polymer blend based DSA (right).

The combination of $\mathrm{CD}$ shrink and CDU improvement offers potential for this type of DSA process to enhance some of the critical items for EUV contact hole patterning. Printing small holes at low dose (required for high throughput) with good CDU is one of the major challenges for this technology [31]. When a negative tone resist is 
A

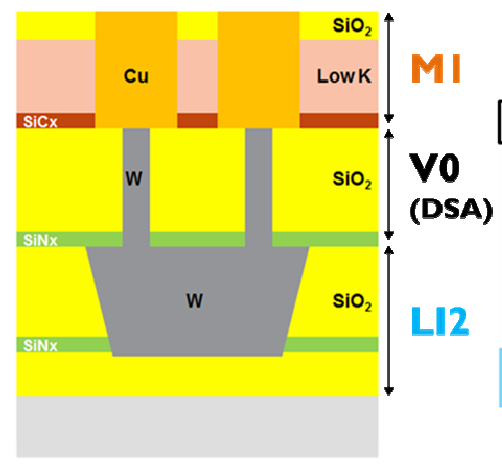

B

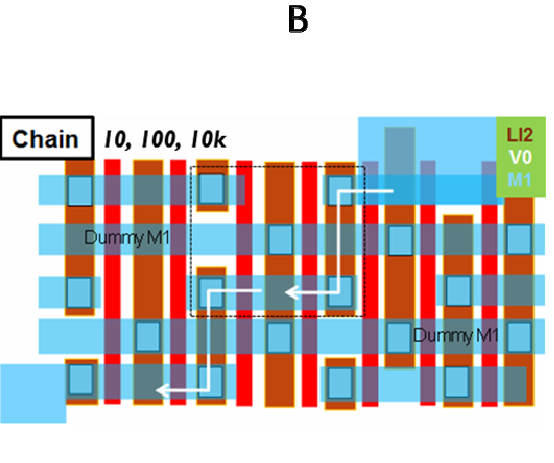

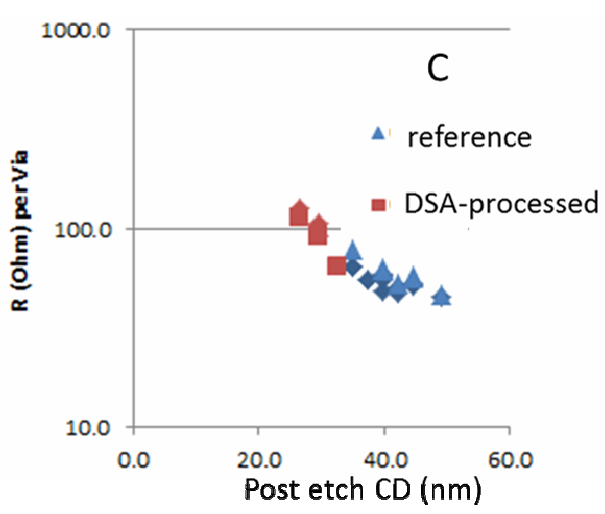

Figure 14. Cross-sectional (A) and top-down (B) view of the EVEREST28 via chain module. Resistivity in the via chains is a function of the via $\mathrm{CD}(\mathrm{C}$; here results for the 10,000 via chain length are given).

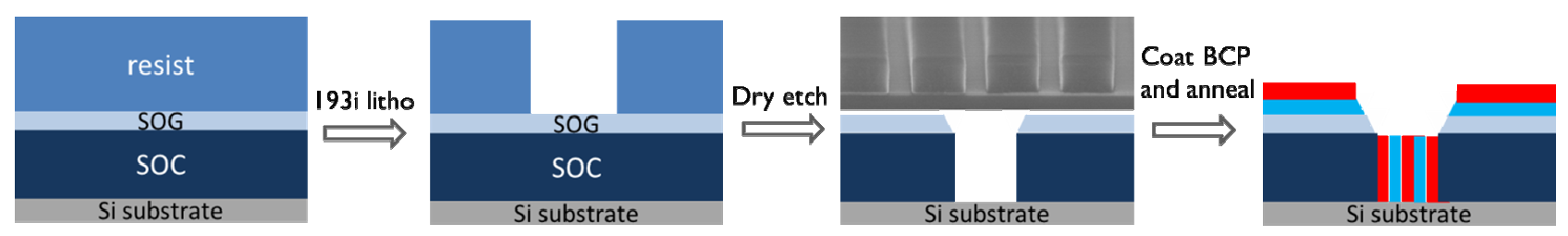

Figure 15. Schematic description of the 1000ISLANDS grapho-epitaxy process flow for hole frequency multiplication. The cross-section SEM inset shows the SOC profile after pattern transfer. Typically, wet development is used to selectively remove the PMMA cylinders.

used, a low exposure dose may be used to print holes with a relatively large CD and 'poor' CDU. Homo-polymer blend based DSA can be used to correct for the $\mathrm{CD}$ and simultaneously repair the CDU (Figure 13). A significantly higher dose is required to target similar $\mathrm{CD}$ and $\mathrm{CDU}$ with the conventional approach using the same resist material [32]. Nevertheless, negative tone resist materials for EUV currently are at a less advanced stage of development compared to the positive tone materials. Therefore, similar performance in terms of dose, $\mathrm{CD}$ and CDU can currently be obtained using a regular positive tone resist material [33]. When better optimized negative tone EUV resist materials become available, this type of approach again may become attractive.

At imec, we also have applied the homopolymer based shrink to assess the potential of DSA to yield electrically functional structures. For this, the $28 \mathrm{~nm}$ logic node compatible via chain short loop has been used (Figure 14A and 14B). In this flow the Local Interconnect 2 (LI2) layer is running perpendicular to an overlaying Metal 1 (M1) layer. At selected intersections, the LI2 and M1 layer are connected by the intermediate Via 0 (V0) layer. This latter layer is treated with homopolymer blend based DSA to shrink CD. Typically, in the reference process a post-litho $\mathrm{CD}$ of $\sim 57 \mathrm{~nm}$ is targeted, which shrinks to $\sim 40 \mathrm{~nm}$ after etch. In the DSA process, the same initial $57 \mathrm{~nm}$ hole shrinks to $\sim 38 \mathrm{~nm}$ after homo-polymer blend treatment and to $\sim 26 \mathrm{~nm}$ after etch. Test structures of varying chain length $(1,10,100$ or 10,000 vias) are available. An assessment has been made of the electrical performance of these structures and the DSA treated results were compared to the reference process where no DSA was used (Figure 14C). The results demonstrate that the higher resistance that was observed from the DSA process can be fully explained by the smaller via CD from this process [34]. When it comes to electrical performance, no intrinsic issues are seen that are induced by the DSA process. Initial efforts to run a similar DSA process based on BCP materials has been started. The DSA-based shrink in this case is more aggressive resulting in 15-18nm CD. Pattern transfer and metal filling optimization at these dimensions are ongoing.

Homo-polymer blends are versatile materials for hole shrink, but are not applicable for hole frequency multiplication. Grapho-epitaxy based frequency multiplication of holes structures is expected to offer a moderate degree of design freedom and, therefore, has been proposed as one of the primary targets for introducing DSA into manufacturing for either contact hole layers [35] or metal cut [36]. At imec this approach is being implemented using a spin-on carbon (SOC) / spin- 


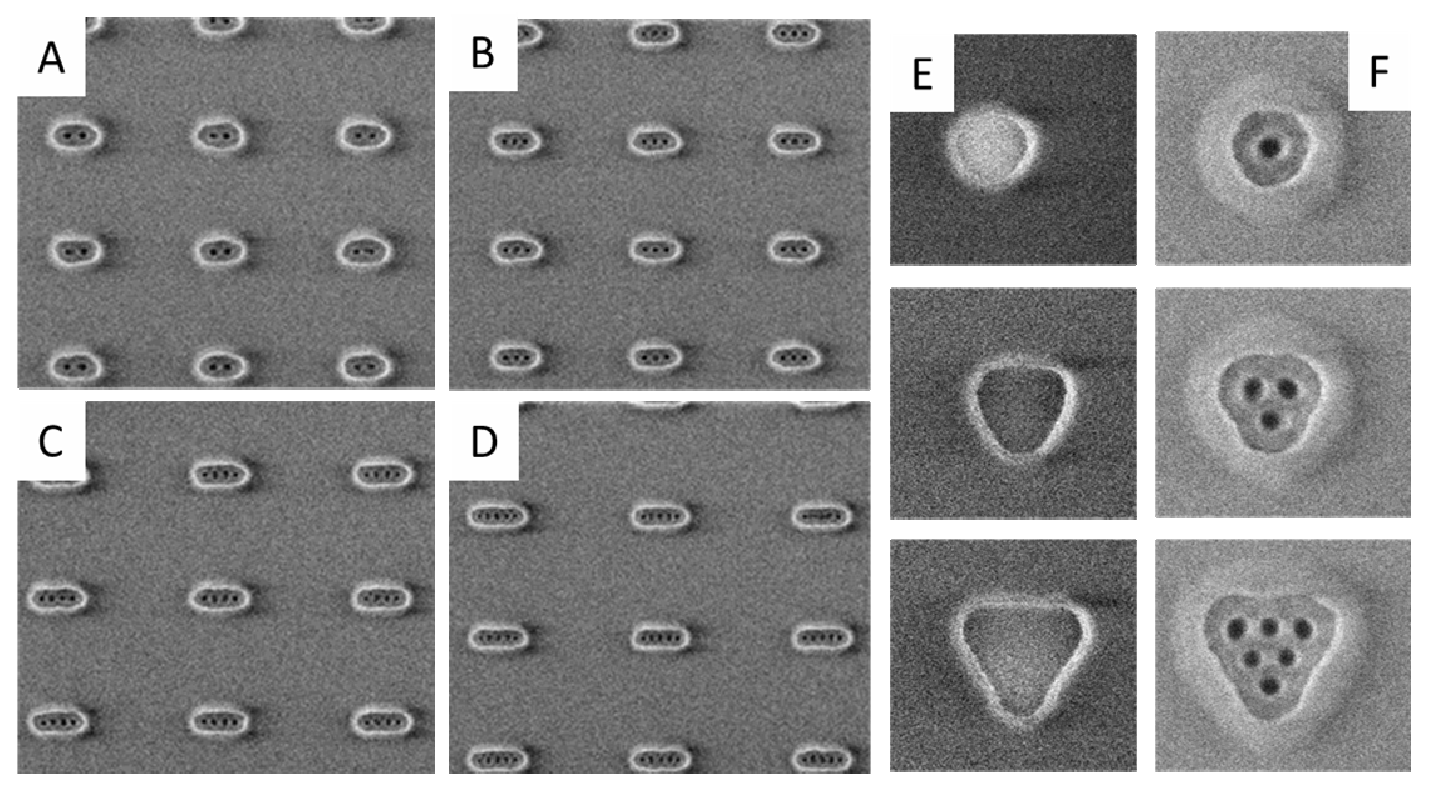

Figure 16. Hole frequency multiplication with the 1000ISLANDS process flow may result in 2 (A), 3 (B), 4 (C), or 5 (D) cylinders per pre-pattern trench. The number of holes per trench is highly reproducible. Triangular shaped prepatterns (E; here top-down images of pre-patterns etched in SOC are shown) may result in 1, 3 or 6 cylinders, depending on the pre-pattern dimension $(\mathrm{F})$.
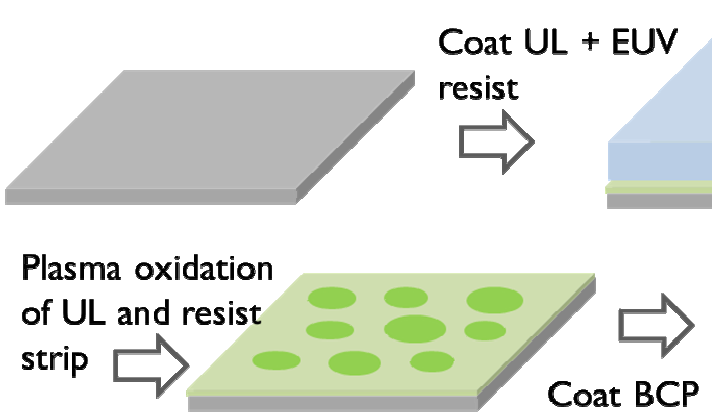
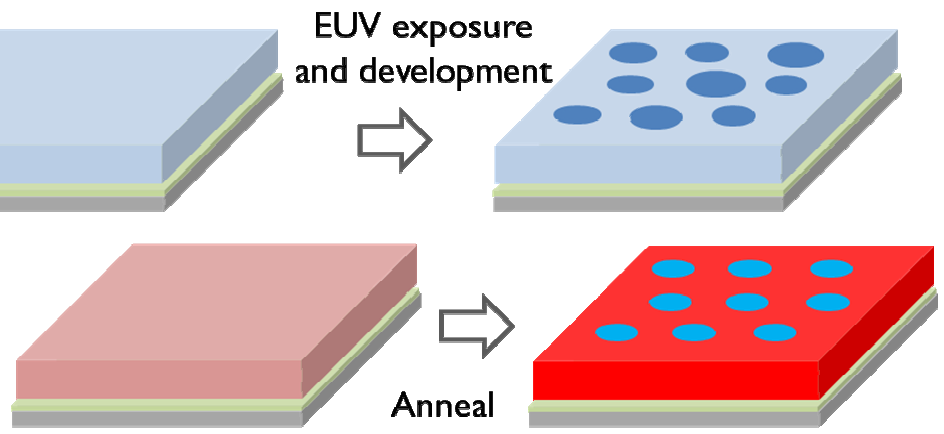

Figure 17. Schematic description of the HONEYCOMB chemo-epitaxy process flow.

on glass (SOG) hard mask stack [37]. This is patterned using a negative tone resist process and $193 \mathrm{~nm}$ immersion lithography (Figure 15). A cylindrical phase BCP material with $37 \mathrm{~nm}$ centerto-center distance is coated over the thus obtained structures and annealed. Depending on the aspect ratio of the short trench pre-patterns, the number of holes in the trench may vary from 2 to 5 (Figure 16A-D). Alternatively, triangular shaped prepatterns can be used to obtain 3 or 6 holes in triangular orientation (Figure 16E-F). The 3 hole triangle is especially interesting, since it is a feature that is frequently encountered in via patterns for logic applications. When the holes are at sub-resolution distance, they currently need to be patterned using 3 individual lithography steps (and masks). DSA processing may simplify this to only a single lithography step.

\subsection{Chemo-epitaxy of hole patterns}

Chemo-epitaxy of cylindrical phase BCP materials allows for formation of hexagonally packed hole arrays. Such hexagonal arrays are especially useful in DRAM manufacturing. Initial efforts have focused on improving CDU of EUV exposed patterns. The HONEYCOMB chemoepitaxy process flow (Figure 17) uses a regular positive tone EUV resist followed by dry etch to modify the surface energy of an underlying surface treatment layer. The surface energy of the untreated material is matched to the majority PS block of the BCP. The dry etch process renders it more hydrophilic and is thus better wet by the minority PMMA block. After stripping of the photoresist, the BCP is coated, annealed and the PMMA block is selectively removed by dry or wet development. 

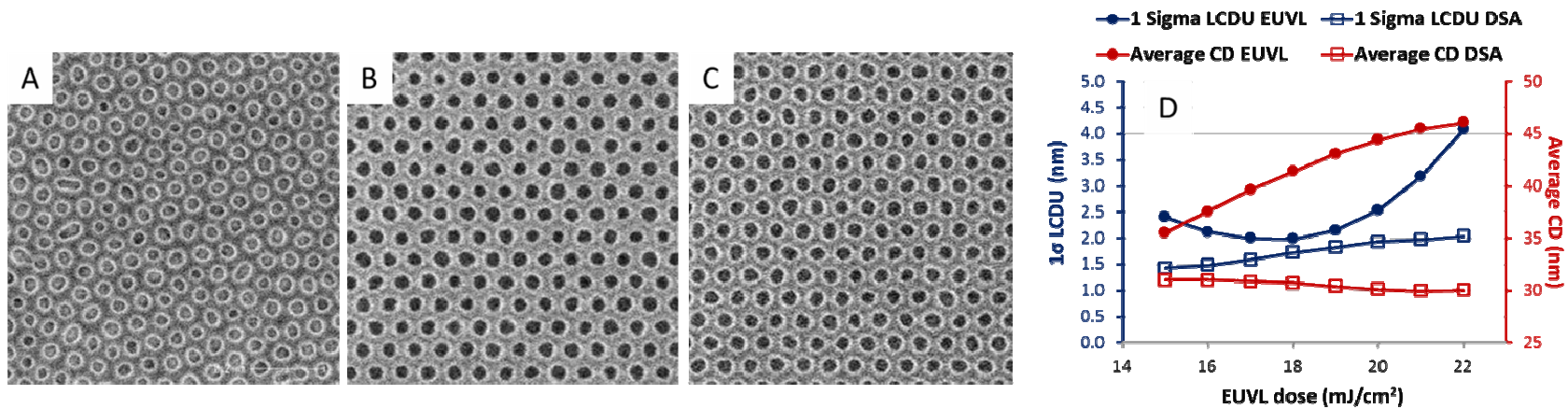

Figure 18. Unguided BCP of $a \sim 58 \mathrm{~nm}$ on neutral substrate (A) shows poor hole-to-hole CD uniformity. Nevertheless, it is able to repair a $58 \mathrm{~nm}$ pitch EUV pre-pattern $(\mathrm{B})$ with $\mathrm{CDU}=2.3 \mathrm{~nm}(1 \sigma)$ to $1.4 \mathrm{~nm}(1 \sigma)$ after the DSA process $(C)$. The post-DSA CD and CDU is fairly insensitive to CD and CDU of the EUV pre-pattern (D).

Initial focus of the HONEYCOMB process flow has been on CDU improvement without frequency multiplication (1:1 patterning) [33]. The resolution limit for hexagonal hole arrays on the ASML NXE:3100 at imec is at $\sim 54 \mathrm{~nm}$ pitch. However, it has been found that the undirected patterns that the BCP provides at these dimensions on neutral layers are irregular and lack the expected hexagonal packing (Figure 18A). The $54 \mathrm{~nm}$ center-to-center pitch is on the high side for BCP materials. When the same BCP, however, is put on a commensurate pre-pattern (Figure 18B) it is able to improve the initial non-uniformities in the pre-pattern from $\sim 2.1 \mathrm{~nm} 1 \sigma$ to $\sim 1.5 \mathrm{~nm} 1 \sigma$ (Figure 18C). The final CDU of the hexagonal hole pattern is quite independent of pre-pattern $\mathrm{CD}$ and CDU (Figure 18D) [37].

Moreover, this approach may be used to restore missing contacts. Missing contacts are expected in EUV lithography due to shot noise effects and mask multi-layer defects. Programmed missing holes on our test structures are repaired with high fidelity (Figure 19) [37].

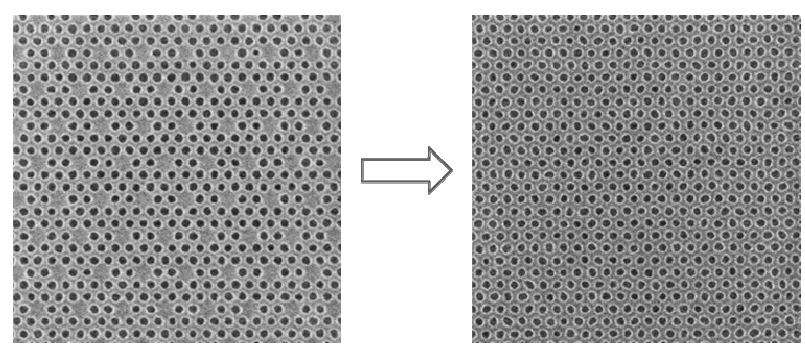

Figure 19. Programmed missing holes in the EUV prepattern (left) are repaired with high fidelity in the HONEYCOMB flow (right).

When the same process flow as described in Figure 17 is used with a BCP that has a cylinder center-to-center distance of $p / \sqrt{3}$ or $p / 2$ (where $p$ is
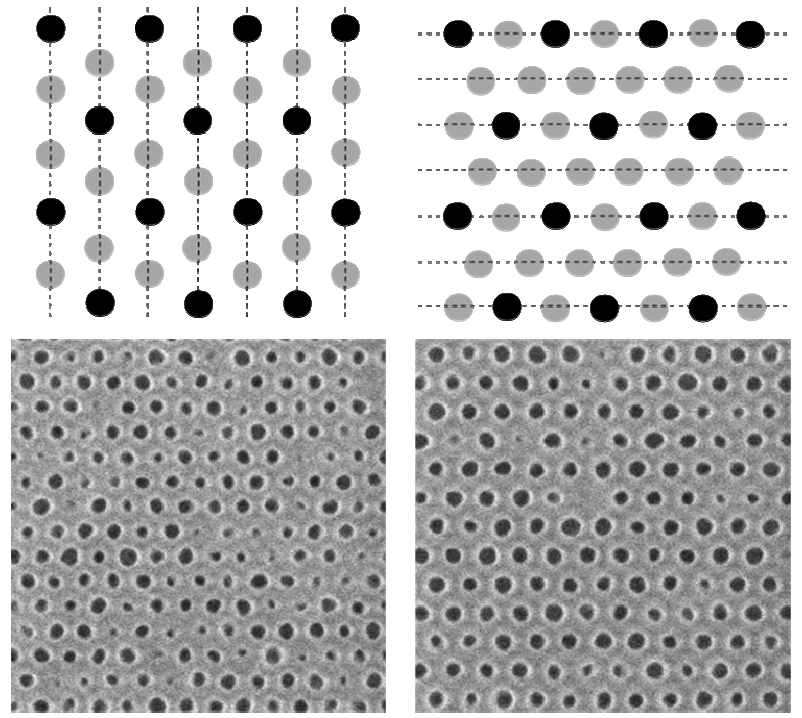

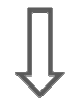
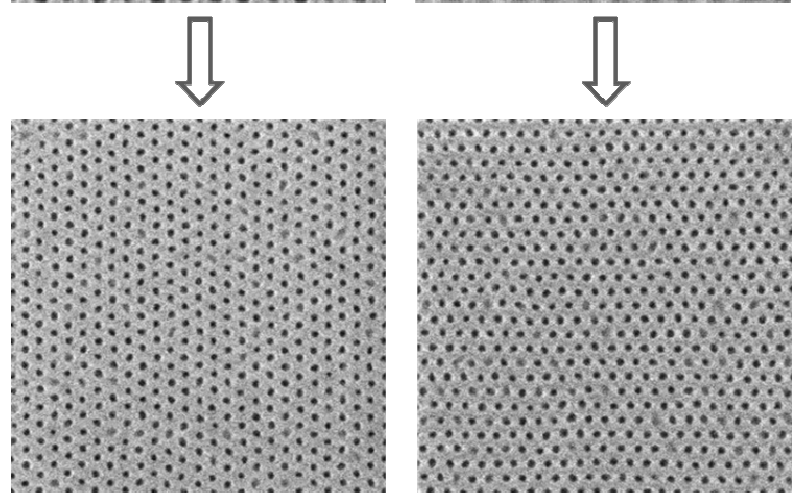

Figure 20. $3 \mathrm{X}$ (left) and $4 \mathrm{X}$ (right) frequency multiplication using the HONEYCOMB flow. Starting from the same hexagonal pre-pattern (black dots in top), $3 \mathrm{X}$ frequency multiplication results in a final pattern (black + gray dots) that is $90^{\circ}$ rotated, compared to the original symmetry axis. Whereas the pattern symmetry is unchanged for the $4 \mathrm{X}$ multiplication. Patterning results (bottom) for a cylindrical phase BCP with $a=$ $30 \mathrm{~nm}$ using a $52 \mathrm{~nm}$ (middle, left) and $60 \mathrm{~nm}$ (middle, right) pre-pattern confirm this.

the pitch of the pre-pattern) $3 \mathrm{X}$ or $4 \mathrm{X}$ frequency multiplication may be expected, respectively 
(Figure 20, top). It should be noted that the $3 \mathrm{X}$ frequency multiplication scheme rotates the axis of symmetry of the pattern by $90^{\circ}$. With the $4 \mathrm{X}$ frequency multiplication scheme, the axis is unchanged. This is confirmed experimentally using a $\mathrm{BCP}$ with $30 \mathrm{~nm}$ natural center-to-center distance and using a $54 \mathrm{~nm}$ and $60 \mathrm{~nm}$ pre-pattern (Figure 20, bottom). The resulting CDU performance from frequency multiplication is much improved compared to the 1:1 patterning, due to the better intrinsic performance of the $30 \mathrm{~nm} \mathrm{BCP}$.

\section{Conclusions}

DSA is a rapidly maturing technology that may find implementation using many different approaches in future patterning technologies. Rather than a disruptive lithographic technology, DSA should be seen as an enhancement technique for traditional (optical) lithography. Its main merits are in pattern rectification/repair and frequency multiplication.

Process flows have been implemented at imec for hole as well as line/space patterning and using grapho- as well as chemo-epitaxy for guiding the BCP structures. Therefore, imec is fully set up to investigate the critical aspects for implementing SA: defectivity, placement accuracy, DSA-specific metrology, and DSA-friendly design decomposition. Finally, electrical demonstration of functional devices that have been patterned with DSA is targeted.

\section{Acknowledgments}

The authors would like to thank the contributing equipment (ASML, Entegris, Hitachi, KLA-Tencor, and Tokyo Electron) and material suppliers (AZ Electronic Materials, Brewer, JSR, and TOK) to the DSA program at imec. Specifically, the on-site assignees are acknowledged for their support.

\section{References}

1. W. Hinsberg, J. Cheng, H.-C. Kim, D. P. Sanders Proc. SPIE 7637 (2010) 7637-0G.

2. K. Lai, C.-C. Liu, J. Pitera, D. J. Dechene, A. Schepis, J. Abdallah, H. Tsai, M. Guillorn, J. Cheng, G. Doerk, M. Tjio, C. Rettner, O. Odesanya, M. Ozlem, N. Lafferty Proc. SPIE 8683 (2013) 8683-04.

3. L. Wan, R. Ruiz, H. Gao, K. C. Patel, J. Lille, G. Zeltzer, E. A. Dobisz, A. Bogdanov, P. F. Nealey, T. R. Albrecht J. Micro/Nanolith. MEMS MOEMS 11 (2012) 031405.
4. L. Leibler Macromolecules 13 (1980) 1602.

5. F. S. Bates, G. H. Fredrickson Annu. Rev. Phys. Chem. 41 (1990) 525.

6. C. A. Ross, Y. S. Jung, V. P. Chuang, J. G. Son, K. W. Gotrik, R. A. Mickiewicz, J. K. W. Yang, J. B. Chang, K. K. Berggren, J. Gwyther, I. Manners Proc. SPIE 7637 (2010) 7637-0H.

7. S.O. Kim, H. H. Solak, M. P. Stoykovich, N. J. Ferrier, J. J. De Pablo, P. F. Nealey Nature 424 (2003) 411.

8. R. A. Segalman, H. Yokoyama, E. J. Kramer Adv. Mater. 13 (2001) 1152.

9. J. Y. Cheng, C. A. Ross, E. L. Thomas, H. I. Smith, G. J. Vansco Appl. Phys. Lett. 81 (2002) 3657.

10. J. Y. Cheng, C. T. Rettner, D. P. Sanders, H.-C. Kim, W. D. Hinsberg Adv. Mater. 20 (2008) 3155.

11. J. Y. Cheng, D. P. Sanders, H.-D. Truong, S. Harrer, A. Friz, S. Holmes, M. Colburn, W. D. Hinsberg ACS Nano 4 (2010) 4815.

12. R. Ruiz, H. Kang, F. A. Detcheverry, E. Dobisz, D. S. Kercher, T. R. Albrecht, J. J. de Pablo, P. F. Nealey Science 321 (2008) 936.

13. S. Park, D. H. Lee, J. Xu, B. Kim, S. W. Hong, U. Jeong, T. Xu, T. P. Russell Science 323 (2009) 1030.

14. T. P. Russell, R. P. Hjelm, P. A. Seeger, Macromolecules 23 (1990) 890.

15. C. M. Bates, T. Seshimo, M. J. Maher, W. J. Durand, J. D. Cushen, L. M. Dean, G. Blachut, C. J. Ellison, C. G. Willson Science 338 (2012) 775.

16. C. B. Tang, S. M. Hur, B. C. Stahl, K. Sivanandan, M. Dimitriou, E. Pressly, G. H. Fredrickson, E. J. Kramer, C. J. Hawker Macromolecules 43 (2010) 2880.

17. G. S. W. Craig, P. F. Nealey Proc. SPIE 7637 (2010) 7637-0L.

18. S.-W. Chang, J. P. Evans, S. Ge, V. V. Ginzburg, J. W. Kramer, B. Landes, C. Lee, G. Meyers, D. J. Murray, J. Park, R. Sharma, P. Trefonas, J. D. Weinhold, J. Zhang, P. D. Hustad Proc. SPIE 8680 (2013) 8680-0F.

19. M. Isawa, K. Sakaia, P. A. Rincon Delgadillo, R. Gronheid, H. Yoshida Proc. SPIE 8681 (2013) 8681-14.

20. R. Gronheid, P. A. Rincon Delgadillo, T. R. Younkin, I. Pollentier, M. Somervell, J. S. Hooge, K. Nafus, P. F. Nealey J. Micro/Nanolith. MEMS MOEMS 11 (2012) 031303.

21. C.-C. Liu, C. J. Thode, P. A. Rincon Delgadillo, G. S. W. Craig, P. F. Nealey, R. Gronheid J. Vac. Sci. Technol. B 29 (2011) 06F203. 
22. P. Rincon Delgadillo, G. Craig, R. Gronheid, P. F. Nealey - published elsewhere in this issue.

23. P. A. Rincon Delgadillo, R. Gronheid, C. J. Thode, H. P. Wu, Y. Cao, M. Neisser, M. Somervell, K. Nafus, P. F. Nealey $J$. Micro/Nanolith. MEMS MOEMS 11 (2012) 031302.

24. P. Rincon Delgadillo, R. Gronheid, C. Thode, H. Wu, Y. Cao, M. Somervell, K. Nafus, P. Nealey Proc. SPIE 8323 (2012) 83230D.

25. P. A. Rincon Delgadillo, R. Gronheid, C. J. Thode, H. Wu, Y. Cao, G. Lin, M. Somervell, K. Nafus, P. F. Nealey J. Photopolym. Sci. Technol. 25 (2012) 77.

26. P. A. Rincon Delgadillo, R. Gronheid, G. Lin, Y. Cao, A. Romo, M. Somervell, K. Nafus, P. F. Nealey Proc. SPIE 8680 (2013) 8680-53.

27. R. Gronheid, L. Van Look, P. Rincon Delgadillo, I. Pollentier, Y. Cao, G. Lin, P. F. Nealey J. Photopolym. Sci. Technol. 26 (2013) 147. 28. Y. Cao, Y. Her, P. Rincon Delgadillo, N. Vandenbroeck, R. Gronheid, B. T. Chan, Y. Hashimoto, A. Romo, M. Somervell, K. Nafus, P. F. Nealey Proc. SPIE 8680 (2013) 8680-1S.

29. P. Rincon Delgadillo, M. Suri, S. Durant, A. Cross, V. R. Nagaswami, D. Van Den Heuvel, R. Gronheid, P. Nealey J. Micro/Nanolith. MEMS MOEMS 12 (2013) 031112.

30. K. Maruyama, Y. Hishiro, R. Imoto, M. Shimizu, T. Kimura "Novel EUV Resist Materials and Process for $16 \mathrm{~nm}$ Half Pitch and Beyond"
2012 International Symposium on Extreme Ultraviolet Lithography Sept 30 - Oct. 4, 2012, Brussels, Belgium.

31. R. Gronheid, G Winroth, A. Vaglio Pret, T. R. Younkin Proc. SPIE 8322 (2012) 8322-0M.

32. T. R. Younkin, G. Winroth, R. Gronheid "A Comparison of Positive- and Negative-tone Contact Hole Process Flows Using the IMEC NXE:3100" 2012 International Symposium on Extreme Ultraviolet Lithography Sept 30 - Oct. 4, 2012, Brussels, Belgium.

33. R. Gronheid, A. Singh, T. R. Younkin, P. Rincon Delgadillo, P. Nealey, B. T. Chan, K. Nafus, A. Romo Negreira, M. Somervell Proc. SPIE 8682 (2013) 8682-0A.

34. T. R. Younkin, R. Gronheid, P. Rincon Delgadillo, B. T. Chan, N. Vandenbroeck, S. Demuynck, A. Romo-Negreira, D. Parnell, K. Nafus, S. Tahara, M. Somervell Proc. SPIE 8682 (2013) 8682-0L.

35. H.-S. Wong, C. Bencher, H. Yi, X.-Y. Bao, L.W. Chang Proc. SPIE 8323 (2012) 8323-03.

36. Y. Borodovsky "Complementary Lithography at Insertion and Beyond" Semicon West 2012, June 11, 2012, San Francisco, CA, USA.

37. R. Gronheid, A. Singh, J. Bekaert, T. Younkin, S. Sayan, N. Vandenbroeck, B. T. Chan, G. Vandenberghe "DSA as a complementary technique for contact hole patterning" 2013 International Symposium on Extreme Ultraviolet Lithography Oct. 4, 2013, Toyama, Japan. 